\title{
HUMOR AND METAPHORS IN MEDICAL LANGUAGE
}

\author{
Monika Gyuró
}

\begin{abstract}
The study makes an attempt to analyze cognitive models of metaphor and humor developed by Fauconnier and Turner (1994) and Attardo and Raskin (1991). Although metaphor and humor are different categories, their cognitive models demonstrate similarities which may create a joint category, metaphorical humor. The study focuses on the co-existence of metaphorical and humorous characteristics in a single expression taken from medical slang. Similarities between the metaphor and humor can be found in the analogies and implications between the contrasting conceptual domains. The difference between the metaphor and humor lies only in the weights of between- and within-domain relationships (Hillson \& Martin 1994). The aim of the paper is to show that the existence of humor and metaphor in a single phrase may reveal common cognitive processes in the creation of metaphorical humor.
\end{abstract}

\section{Keywords}

humor, metaphor, cognitive processes, cognitive models, medical language, medical slang

\section{Introduction}

Until the twentieth century, the metaphor was essentially considered as a trope belonging solely to poetry. Cognitive linguistics (CL) within cognitive psychology demonstrated a new perspective to scholars in understanding how language is used. CL focuses on language in terms of concepts, i.e. the underlying mechanisms of their evolution which may provide explanation about the essence of the metaphor. The paradigm change began in the seminal work of Lakoff and Johnson (1980), who stated how we use metaphors in a broad sense in which meanings of single words can be tools which we use to conceptualize less clearly understood terms with the help of more clearly conceived ones. Thus, metaphorical thinking has been extended to cognition in which concepts are being formed in association with other concepts. In "The Conceptual Mapping Theory (CMT)", Lakoff and Johnson (1980) suggest our understanding of words is based on finding connections between different domains of concepts. On the one hand, source domain is a conceptual domain which organizes the unknown in terms of the known. On the other hand, however, target domain is a conceptual domain which is understood metaphorically in terms of the source domain. A particular version of CMT included an approach which investigated the distance 
in between- and within-domain positions. Tourangeau and Sternberg (1982) made a remarkable observation in which the constituents in metaphors must have a long distance between the domains and short distance in within-domain positions.

The approaches mentioned above involve a common feature in which the implementation and use of identical properties between concepts enables readers to understand the unknown concept in terms of the known. However, the dominant CMT emphasizes a stable relationship between the domains in the use of a metaphor whereby the structure is positioned together in mapping, concerning the background knowledge of the speakers. A modern version of metaphor theory attempted to provide a more dynamic model of figurative language comprehension, which focused on the process of comprehension and inference of concepts in a four-space model. Fauconnier and Turner (1994) claim the basic unit in metaphor structure is not the domain, but the mental space speakers construct with regard to a situation in life. These spaces involve an 'input' and an 'output' space, corresponding with target and source domains in Conceptual Blending Theory (CBT), a 'generic space' which is a conceptual structure of both aforementioned spaces, and the 'blend space' where attributes from input and output blend. The latter space accounts for inference receiving information from the former spaces and develops a new, logically relevant structure based on but not available from the input and output. Consequently, in CBT, elements from diverse scenarios which are inevitable to everyday knowledge and language use are blended.

In comparing metaphors to humor, humor shares common features with metaphors such as the dual planes of meaning, a semantic contradiction, and the bisociativity between the two frames of reference (Krikman 2009). Investigating the literature of humor in detail, in Arthur Koestler's tract (1964) human thinking is determined by bisociativity which is a specific two-planed creative act. In humorous texts, this means an oscillation between two worlds of discourse. The idea was further developed by Greimas (1971), who focused on the basic concepts of general semantics showing that the same expression can have ambiguous readings resulting in figurative or humorous language. The notion of isotopy became a central issue in the theory of linguistic humor. Isotopy means different interpretations of ambiguous expressions and text; therefore, we must find a process of disambiguation to reveal the meaning of the discourse. This idea was elaborated further by Raskin and Attardo (1991). Their approach (Semantic Script-Based Theory of Humor) presented the knowledge structures of the listeners by which they can process humor in the form of a joke. 
In conclusion, both metaphors and humor apply split reference when they concern two different but related images or scripts. Hillson and Martin (1994) combined the incongruity-resolution theory of humor with the approach of between- and within-domain relationships developed by Tourangeau and Sternberg (1982) in support of metaphors. It was found in reference to metaphors that the criterion of short within-domain distance (similarity) possessed relatively greater weight. However, when considering jokes, the long betweendomain distance (incongruity) outweighed short within-domain distances. It is worth mentioning yet another important theory of humor which may explain the function of humorous texts. The Superiority Theory (ST) is a social approach which determines humor as an expression of misfortunes. In the framework of this theory, Plato (1989) determined humor as an attack on personal weakness, while Aristotle (2004) explained how laughter originates from humiliating the person through interaction.

Despite the numerous studies published in the fields of metaphor and humor (Plato 1989, Aristotle 2004, Lakoff \& Johnson 1980, Fauconnier \& Turner 1994, Tourengeau \& Sternberg 1982, Raskin \& Attardo 1991, Hillson \& Martin 1994), analysis of their common use in a single phrase remains a major challenge for scholars. Thus, the starting point of the study was the recognition that a great number of phrases in medical slang are characterized by metaphorical humor. The study postulates that the co-existence of the metaphor and humor in a single expression may demonstrate their adjacent cognitive origin. The primary objective of the study is to analyze metaphor and humor comprehension in a single phrase borrowed from medical slang in order to demonstrate the underlying cognition in support of them. A combined approach of SSTH, CBT and ST is used in the analysis to describe the cognitive processes which may explain the potential functioning of metaphors and humor in a single expression. To date, this issue has not been researched extensively within cognitive linguistics.

\section{Corpus and method}

The language data of medical slang discussed in this study is taken from an internet-based website entitled, Doctors' Slang, Medical Slang and Medical Acronyms and Veterinary Acronyms and Vet Slang (http://messybeast.com/ dragonqueen/medical-acronyms.htm). The corpus amounts to 916 words, phrases, and acronyms derived from the practitioners in the US, UK, and Australia. Although relatively small in size, the specialized corpus described above is considered to be sufficient and adequate for the present analysis, since the representativeness of the terminology can be seen from the data sources and quantity. Considering the present investigation, 95 metaphors were found in the 
database from which I selected only two figures to be analyzed. The corpus of a doctor's joke originates from a collection of medical jokes (Gyuró 1994).

Based on the definition of the Merriam-Webster's Collegiate Dictionary (2003), slang is “... an informal or non-standard vocabulary composed of coinages, arbitrarily changed words, and extravagant or forced figures in speech." A special branch of slang is medical slang which is commonly prevalent among medical students. Medical slang involves a specific vocabulary which has not been formalized in any medical text, yet is almost understood by nearly all junior physicians. The use increases during the last medical student years and peaks during internship when responsibilities are the most strenuous (Fox et al. 2003). Derogatory humor may serve important functions, such as the survival and wellbeing of the professional within a set of challenging circumstances. It helps the professional distance oneself from disease and death. Medical slang is thought to overcome the difficulties in medical practice, such as the long hours of study, tiredness, and communication with patients. Medical slang is characterized by cynicism which targets mainly particular groups of patients whose health problems are perceived to be controversial. These groups are considered as 'problem patients' due to their behaviors which may inhibit professionals to take care of them. The terms are not used in front of the patients because they demonstrate frustration against the clients. The sources of medical slang can be various. Most slang terms can be learnt from seniors, but there are many other sources of terminology. Publications, such as Keating's (1993) Bluffer's Guide, and Mercurio's (2002) Bodies are worth mentioning. Taking the lexical forms of various slang terms into consideration, Dirckx's (1983) classification seems to be essential, in which medical slang is characterized by a great number of short words, the use of acronyms, and metaphors. Metaphors and humor often occur in the same word or phrase, such as in "red pipe" referring to a blood vessel.

Regarding the method used for analyzing metaphors in the corpus, the CBT developed by Fauconnier and Turner (1994) was applied. According to this theory, elements from diverse scenarios are combined into an integrated mental space which develops an emergent structure not available from other inputs. As for the methods used in the analysis of humor, the Semantic Script-Based Theory of Humor was applied. SSTH as an incongruity-resolution theory (Raskin 1985, Attardo \& Raskin 1991) in which it is claimed a joke can be represented as a script with references to a real life situation. The script involves two possible interpretations which are opposed to one another. There are three types of oppositions, such as actual/non-actual, normal/abnormal, possible/impossible dualities. These are classified as more specific oppositions, such as good/bad variations. Consequently, the oppositions exhibit varying degrees of generality. 
The processor realizes the duality and the tension between the scenarios resulting in laughter. Additionally, I applied the Superiority Theory (Aristotle and Plato) to complement the findings of SSTH. The theory above (ST) claims the source of humor is the unexpected surprise. Surprise originates from an opposition between what is expected and what is actually realized. The latter theory demonstrates humor as a personal weakness or a pleasure to belittle someone.

The purpose of the present analysis is to demonstrate a cognitive approach to metaphorical humor; therefore, a cognitive model is proposed which demonstrates the properties of both SSTH and CBT complemented by the Superiority Theory. This model of metaphorical humor was first introduced by Kitazume (2006), who explained metaphor and humor of contrasting and analogical domains. The examples of the present study were taken from medical slang which is a rich source of metaphorical humor. The data was examined and processed manually in order to obtain qualitative results and interpreted with the help of the cognitive models above.

\section{The Conceptual Blending Theory of the metaphor}

According to CBT, the metaphor involves four planes of meaning including a semantic opposition between the input and output spaces, a generic space involving the analogies between the aforementioned two spaces, and an additional blend space which carries the inference based on the input and output. The output space refers to a literal meaning to be explained by the expression used metaphorically in the input space. The generic space forms the basis, as a third space for involving analogies between the input and output (Fauconnier \& Turner 1994).

I attempt to illustrate a cognitive model of the metaphor through a medical slang expression. The corpus of medical slang involves several metaphors, such as "banana" (a patient with jaundice), "bungee jumper" (a patient who pulls on his catheter tube), "death camp" (a terminal nursing home), and "rose cottage" (a term for the mortuary). I selected only one metaphor from the corpus in order to analyze it within the framework of CBT. "House red" refers to blood in the medical context. The Collins English Dictionary (2016) defines the original term above as, "a red wine sold unnamed by a restaurant, at a lower price than wines specified on the list". The cognitive model of the metaphor "house red" describes the output space (OS) on the left and the input space on the right (IS). The output and input sets contain the attributes of the above spaces. Blending is shown by the set-section in the generic space. I implemented a Venn diagram in order to illustrate the blending between the input, output, and generic spaces within CBT. According to my view, the model describes the relationships among 
the mental spaces of a metaphor better than the original figure represented in the theory of Fauconnier and Turner (1994).

$\mathrm{P}=$ Property $\mathrm{G}=\mathrm{Generic}$ space $\mathrm{B}=\mathrm{Blend}$ space

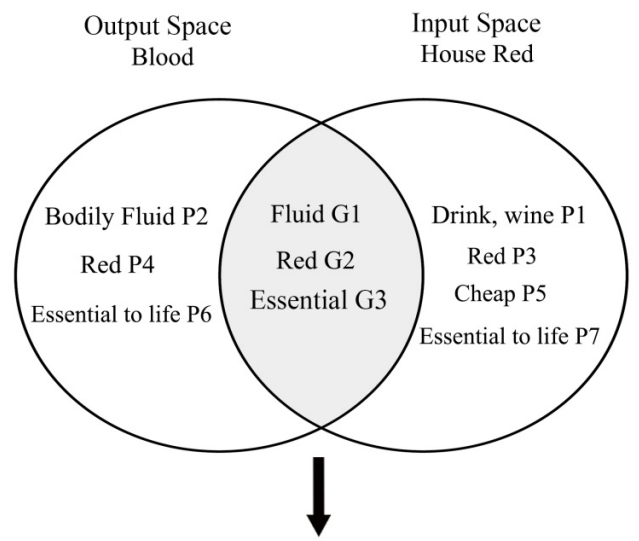

Blend Space

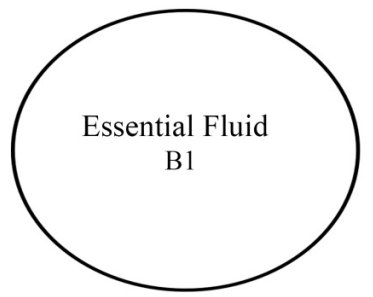

Inferential directions/relationships:

$$
\begin{array}{ll}
\mathrm{P} 1 \rightarrow \mathrm{P} 2 & \mathrm{P} 1 \approx \mathrm{P} 2 \\
\mathrm{P} 3 \rightarrow \mathrm{P} 4 & \mathrm{P} 3=\mathrm{P} 4 \\
\mathrm{P} 5 \rightarrow \mathrm{P} 6 & \mathrm{P} 5 \leftrightarrow \mathrm{P} 6 \\
\mathrm{P} 6 \rightarrow \mathrm{P} 7 & \mathrm{P} 6=\mathrm{P} 7
\end{array}
$$

Analogical inferences:

$\mathrm{P} 1 ; \mathrm{P} 2=\mathrm{G} 1 ; \mathrm{P} 3 ; \mathrm{P} 4=\mathrm{G} 2 ; \quad \mathrm{P} 6 ; \mathrm{P} 7=\mathrm{G} 3$

$\mathrm{P} 1 ; \mathrm{P} 2 ; \mathrm{P} 6 ; \mathrm{P} 7=\mathrm{B} 1$

Figure 1: The cognitive model of a metaphor 
Figure 1 demonstrates the term "blood" in the output space above the left set and the term "house red" in the input space above the right set. I may implicate the term "house red" is based on our background knowledge and the obvious reference to wine. In European cultures, blood is often associated with wine; in liturgy, believers drink wine as a symbol of Christ's blood in order to reunite with him. The properties of the output and input spaces are listed in the sets in each domain. These features are described by a definition in the Collins English Dictionary (2016) and also, that of physiology. The figure demonstrates how the generic space connects the elements in OS and IS in the form of analogies.

The starting point for the inferential process is the input space. P1 illustrates "house red" is a wine which is naturally a fluid. I must mention that the term "house red" is a metaphor itself; therefore, some encyclopedic knowledge is necessary in its processing. P2 indicates how "blood" is a fluid itself compared to the term "house red". However, we can see there is no direct correlation between the two concepts. Wine is not blood despite its identical consistency, but the correlation between the two concepts is quite close. The next step involves finding similarities between the two entities with respect to their color. P3 and P4 highlight identical features. The next property of IS (P5) lies in its inexpensive nature. As we cannot find an analogy in OS for this property, only its contrast is found: P6. P6 in IS implicates if P6 in IS "is essential to life" then P7 should be essential to the implication of $\mathrm{P} 2$. It cannot be different from amusement, as wine is associated with high spirits. The generic space involves analogical elements which links the two contrasting spaces. These are the consistency, color and the value of the concepts (G1, G2 and G3). Processing P1; P3 in OS and P2; P4 in IS is based on common knowledge. The selection of $\mathrm{P} 5$ requires special knowledge on wine, while its inference in P6 originates from basic biological knowledge. In the blend space, the overall inference can be found in the expression, "essential fluid (B1)". This property bears the common features of both the input and the output spaces.

In summary, I claim in-domain properties of the concepts demonstrated short, yet between-domain or space properties of the concepts exhibited shorter distance than it was originally expected.

\section{The SSTH model to humor}

The cognitive model of humor developed by Attardo and Raskin (1991) clearly shows the major structure and evolution of humor used in language.

According to the model above, a joke typically begins initially with an ambiguous initial part, which has two possible interpretations. One part is more dominant while the other is less obvious for the audience. Following 
the processing of the joke, the secondary interpretation becomes obvious. The major elements of a joke involve two opposing interpretations such as normal or abnormal. The joke about the bronchial patient (Raskin 1985: 32) demonstrates this structure. The joke starts with the normal interpretation, but the whisper of the doctor's wife shows a different or abnormal approach of the situation. The reader has to restore the original or normal coherence of the text.

"Is the doctor at home?" the patient asked in his bronchial whisper. "No," the doctor's young and pretty wife whispered in reply. "Come right in."

Let us illustrate humor in the following example (Gyuró 1997: 133) whose effect can be best explained by the cognitive model above in details.

"Did you follow my prescription?"

"No, if I had, I should have broken my neck, because the draught blew it out of the window".

The initial question has two possible interpretations in the example above. The first interpretation or script is based on the normal use of the phrase. The doctor's query targets the patient's compliance with respect to prescription medication and treatment. The second script is fairly impossible or abnormal. The patient understands the doctor's question literally. He conceives the word "follow" in its physical sense which means, of course, "go after". If the wind blows the prescription out of the window, it seems to be difficult to go after it. In the joke, normal and abnormal interpretations of the participants are opposed to one another, resulting in humor.

Figure 2 demonstrates the two scripts as normal and abnormal interpretations of the situation. The contrast between the two different interpretations triggers incongruity in the audience which is the source of humor. The basis of incongruity between the two scripts can be found in the different use of literal and non-literal use of words and phrases in language. A variety of the non-literal use of words and phrases is referred to as idioms consisting of ordinary words with literal meanings, but if they are used in a phrase, the meaning of the utterance is more than the sum of the components (Traxler 2012). There are two main sorts of idioms; non-decomposable and decomposable ones. In reference to Example 2 above, a decomposable idiom ("follow prescription") plays an important role in the joke above. Decomposable idioms can be broken into parts and each part can be related to a component within the idiom's definition. Gibbs et al. (1989) claim decomposable idioms are syntactically flexible as their parts can be substituted 
with synonyms. In processing, idioms are comprehended word by word, thus, independent meanings can be assigned to their parts. This syntactical flexibility emerges as an essential factor in humor comprehension as well, since listeners can easily decompose the non-literal meaning of the idiom into a literal one.

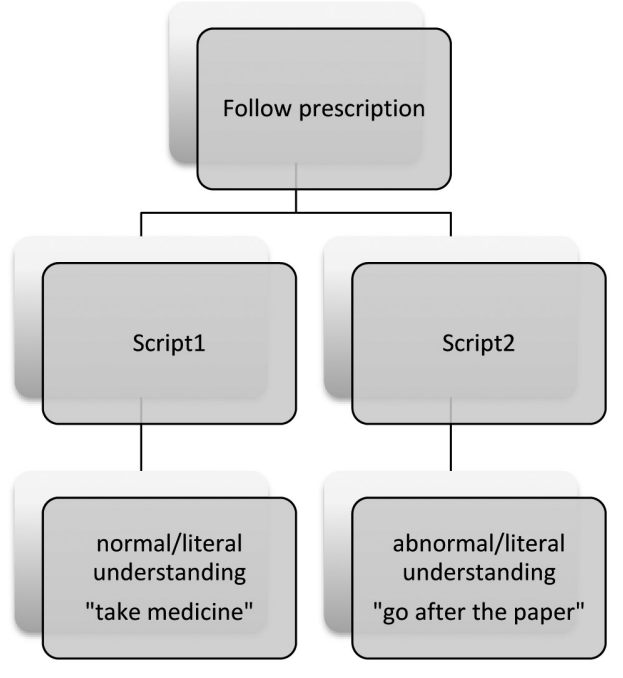

Figure 2: The cognitive model of humor

In the next step of the analysis, I attempted to illustrate the characteristics of metaphorical humor with a joint model of CBT and SSTH.

\section{A cognitive model of metaphorical humor (СМMH)}

Investigating the CBT of the metaphor and the SSTH of humor separately, I attempted to provide a cognitive model which applies the attributes of both CBT and SSTH complemented by the Superiority Theory. Thus, I can describe metaphorical humor showing the resemblance in the process of understanding metaphor and humor within a single phrase.

The proposed cognitive model of metaphorical humor (CMMH) consists of two opposite scripts or spaces and a common generic space that connects the two spaces. Regarding humor, the analogical properties provide illustrative associations between the two scripts. However, the opposing scripts feature a substantial distance between the concepts to be compared. The rule for the $\mathrm{CMMH}$ can be formulated in this way: Analogies in the generic space should be 
as close as possible, but concepts in the input and output spaces require longer distance or more associative steps in their comprehension. A great number of implications are involved in the comprehension process of metaphorical humor. Understanding metaphorical humor requires associative and pragmatic efforts by the audience. Hence, I can conclude that analogies create correlations between two conceptual spaces in the case of metaphorical humor. However, analogical thinking is deemed a necessary but not a sufficient condition to process metaphorical humor. In analyzing the case of humor in CBT, the immense distance between the input and output spaces and the many associative steps between the concepts can often explain incongruity, surprise, and consequently, laughter, as inevitable elements within this genre. Additionally, the importance of the Superiority Theory plays an essential role in the spectrum of the humor found in medical slang. The medical slang phrase analyzed below suggests a sort of cynicism, which attempts to belittle its subject.

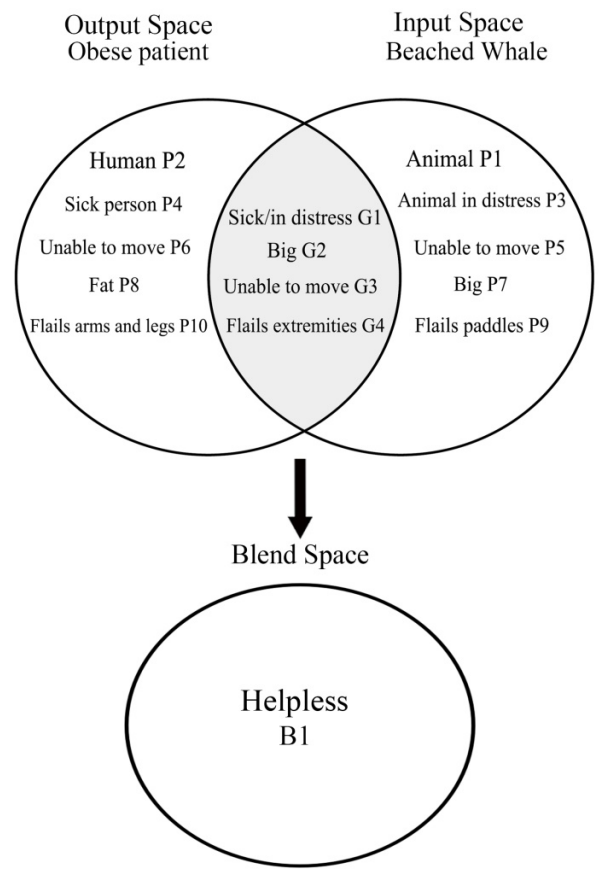


Inferential directions/ relationships:

$\mathrm{P} 1 \rightarrow \mathrm{P} 2 \quad \mathrm{P} 1 \leftrightarrow \mathrm{P} 2$

$\mathrm{P} 3 \rightarrow \mathrm{P} 4 \quad \mathrm{P} 3=\mathrm{P} 4$

$\mathrm{P} 5 \rightarrow \mathrm{P} 6 \quad \mathrm{P} 5=\mathrm{P} 6$

$\mathrm{P} 7 \rightarrow \mathrm{P} 8 \quad \mathrm{P} 7=\mathrm{P} 8$

$\mathrm{P} 9 \rightarrow \mathrm{P} 10 \quad \mathrm{P} 9=\mathrm{P} 10$

Analogical inferences:

P3; P4=G1

$\mathrm{P} 5 ; \mathrm{P} 6=\mathrm{G} 2$

$\mathrm{P} 7 ; \mathrm{P} 8=\mathrm{G} 3$ and $\mathrm{P} 9 ; \mathrm{P} 10=\mathrm{G} 4$;

Figure 3: The cognitive model of metaphorical humor

In the example above, the slang metaphor, "beached whale" refers to an obese patient who is unable to do anything for him or herself, except to lie there with flailing arms and legs (Doctor'slang dictionary). Figure 3 demonstrates how metaphorical humor is construed from three parts. The output space (OS) refers to the obese patient and the input space (IS) is related to the phrase, "beached whale". Analogies between the two spaces are found in the generic space. In this model, the oppositions between the spaces, concepts, their analogies and implications create both metaphorical and humorous thinking in a single phrase. "Beached whale" is the metaphor of an extremely overweight person who cannot move. The common properties in the generic space, such as fat, big, unable to move, flailing extremities, were obtained from the inferential processes during comparison of the two concepts. The starting point is the definition of a "beached whale" in IS. P1 refers to an animal. Then the listener must find an impossible counterpart of the OS with the same properties as in IS. In OS, it is a human being (P2). According to observations, a "beached whale" hardly moves (P3), thus it can be seen in distress and distanced from the sea. By inference, an obese patient is sick and tied to bed (P4). Both of them are unable to move (P5 and P6). The next step in processing is to find another common feature between IS and OS. A large animal (P7) may be the suitable choice, since the counterpart, the obese person, is similarly as big as the animal to be compared (P7). Both of them are unable to generate movement but can flail their extremities (P9 and P10). Analogies between OS and IS may allow us to consider the phrase merely a metaphor. The source of humor lies in the distance between the input and output spaces and the speaker's intention to exaggerate the patient's disabilities. The distance is expressed by the different classification of the two participants: one of 
them is a sick human and the other one is an unfortunate animal. In the analysis and the issue of exaggeration, an obese patient is not as large as a whale, but to make the person appear ridiculous, the speaker must exaggerate the size of the patient in comparison to an extremely huge animal. Exaggeration is an important factor for the speaker to be able to feel superior in the situation. The comparison between an obese patient and a whale is cynical from the point of view of the speaker. This attitude may generate laughter in the audience experiencing incongruity between the two concepts and superiority in the role of the speaker.

In conclusion, I claim that analogies between concepts can be necessary conditions for construing a metaphor. However, these factors are not sufficient in developing humor in the core of language. Humor requires incongruity between the concepts and a sort of superiority from the role of the speaker to be able to attack personal weakness.

\section{Discussion}

The study aimed at detecting the cognition in metaphor and humor processing through examples selected from medical slang. I attempted to justify the hypothesis, in which despite the differences these entities possess, there are common features in their development of human thinking. This idea is supported by my sample, namely medical slang phrases, which demonstrate that metaphorical humor can exist in a single expression. The co-existence of metaphor and humor in a single phrase has not yet been extensively researched.

The analysis of the examples has pointed out the similarities between metaphor and humor suggested by Krikman (2009), such as the dual planes of meaning, the pragmatic contradictions, and the bisociativity between the two frames of reference. The differences between metaphor and humor in our examples supported the findings of Hillson and Martin (1994), who found short within-domain distance in metaphor and long between-domain distance in humor processing. Short within-domain distance refers to analogy and long betweendomain distance is related to incongruity regarding the comprehension process of these entities.

Researchers extensively investigated metaphor and humor separately, either in a joke or in a metaphorical phrase. Kitazume (2006) investigated metaphorical humor in a joke, but not in a single phrase. The findings of the present study demonstrate that metaphorical humor can be found in a single expression showing an oscillation between analogy and incongruity of concepts. This analysis described metaphorical humor in a single expression, within two conceptual spaces, through input and output with generic and blend spaces. OS and IS carried the properties of the two concepts and the generic space referred to the 
analogies between the two spaces. However, OS and IS exhibited a fundamental incongruity of the concepts as the source of humor. The claim of Hillson and Martin (1994) was justified in this way. The blend space showed the dynamic aspect in metaphorical comprehension and described the inferential process in thinking at the same time.

Limitations of the findings in the analysis should be kept in mind. The small size of corpus should be extended in the future analyses and a more profound investigation is needed to demonstrate the similarities and differences in metaphor and humor processing in a single phrase.

\section{References}

Aristotle (2004) Poetics. Chapter 21.Transl. by S. H. Butcher. Adelaide: eBooks.

Attardo, S. and Raskin, V. (1991) 'Script theory revis(it)ed: Joke similarity and joke representation model.' HUMOR: International Journal of Humor Research 4-3/4. 293-347.

Collins English Dictionary (2016) London: Harper Collins Publisher.

Dirckx, J. H. (1983) The Language of Medicine. Santa Barbara, CA: Praeger Publisher. Inc.

Fauconnier, G. and Turner, M. (1994) Conceptual Projection and Middle Spaces. Department of Cognitive Science Technical Report 9401. San Diego: University of California, San Diego.

Fox, A. T., Fertleman, M., Cahill, P. and Palmer, R. D. (2003) 'Medical slang in British hospitals.' Ethics and Behaviour 13/2. 173-189.

Gibbs, R. W. (1994) The Poetics of Mind: Figurative Thought, Language, and Understanding. New York and Melbourne: Cambridge University Press.

Greimas, A. J. (1971) Strukturale Semantik. Metodologische Untersuchungen. Wiesbaden: Vieweg+Teubner Verlag.

Gyuró, M. (1994) Readings on Nutrition for Learners of English. Pécs: Faculty of Health Sciences. Pécs Medical University.

Hillson, T. R. and Martin, R. A. (1994) 'What's so funny about that? The domaininteraction approach as a model of incongruity and resolution in humor.' Motivation and Emotion 18/1. 1-29.

Keating, P. (1993) The Bluffer's Guide to Dictionary: Bluff Your Way in Doctoring. London: Ravette Books.

Kitazume, S. (2006) 'Cognitive models of humor and metaphor.' Literature, Arts, and Cultural Studies 3. Osaka: Kinki University. 125-150.

Koestler, A. (1964) The Act of Creation. London: Hutchinson and Co.

Krikman, A. (2009) 'On the similarity and distinguishability of humour and figurative speech.' TRAMES. Journal of Humanities and Social Sciences 13/1. 14-40.

Lakoff, G. and Johnson, M. (1980) Metaphors We Live By. Chicago-London: Chicago University Press.

Mercurio, J. (2002) Bodies. London: Johnatan Cape.

Merriam-Webster's Collegiate Dictionary (2003) US: Merriam-Webster.

Plato (1989) Philbus. In: Hamilton, E. and Cairns, H. (eds) The Collected Dialogues of Plato. Bollingen Series. Princeton: Princeton University Press. 575-844. 
Raskin, V. (1985) Semantic Mechanisms of Humor. Dordrecht-Boston-Lancaster: D. Reidel Publishing Company.

Tourangeau, R. and Sternberg, R. J. (1982) 'Understanding and appreciating metaphors.' Cognition 11/3. 203-244.

Traxler, M. J. (2011) Introduction to Psycholinguistics. New Jersey: Wiley-Blackwell.

\section{Internet source:}

Doctors' Slang, Medical Slang and Medical Acronyms and Veterinary Acronyms and Vet Slang (http://messybeast.com/dragonqueen/medical-acronyms.htm).11.November 2015 .

Monika Gyuró is Assistant Professor of English Language and Health Communication at the Faculty of Health Sciences, Pécs University, Pécs, Hungary. Her research fields involve discourse analysis, pragmatics and argumentation theory, focusing on medical, legal, and political discourses. She has been participating in the work of the Pécs School of Argumentation since 2003. This inter-university research cooperation funded by the National Science Foundation and the Hungarian Academy of Sciences is linguistically oriented. She investigates discourse coherence and arguments in health-related interviews, in which argumentation theory is applied to the clinical interview.

Address: Monika Gyuró, Ph.D., Institute for Health Insurance, Faculty of Health Sciences, Pécs University, Mária u. 5-7., 7621-Pécs, Hungary. [e-mail: gyuro@etk.pte.hu] 